\title{
Call for Special Issue Papers: The Human Aspects of Cybersecurity
}

\section{Deadline for Manuscript Submission: August 1, 2020}

\author{
Guest Editor: Scott Debb \\ Norfolk State University, The Virginia Consortium Program in Clinical Psychology, Norfolk, VA, USA
}

\begin{abstract}
Social engineering, software exploits, cyber attackers, cyber defenders, and hackers wearing all shades of white and black hats alike all have one common thread: a human element. The human being is at the heart of all that defines cybersecurity. Efforts to secure the global digital landscape often yield inconsistent results, with tools of automation often being deployed side by side with educational initiatives to empower people to be responsible for their cybersecurity needs. There are many large-scale collaborative efforts by social scientists and computer scientists alike to help facilitate safe and trustworthy cyberspaces, yet we regularly hear news reports of widespread data breaches at large institutions, not to mention the myriad of malware and ransomware-related issues affecting large portions of the general population.

There is no shortage of research highlighting the technical aspects of cybersecurity, and there is also an increasing amount of literature that attempts to understand the psychological profile of individuals who either engage in cybersecurity practices or attempt to infiltrate cybersecurity infrastructure. However, there is a need to have a greater understanding and awareness of the human element across all aspects of cybersecurity, specifically research that has the capacity to facilitate discussions that translate across discipline areas, such as computer and social sciences.
\end{abstract}

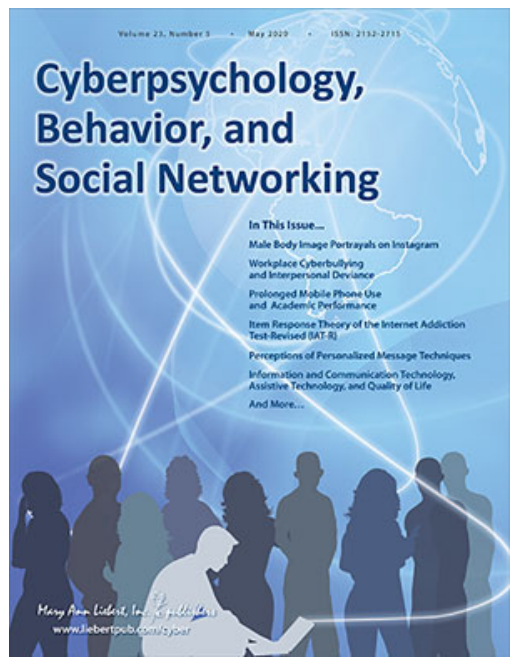

We are seeking original articles that are interdisciplinary in nature and help further psychology's understanding of what the human element in cybersecurity represents. Potential qualitative or quantitative studies focusing on the following topics will be given full consideration for this special issue of Cyberpsychology, Behavior, and Social Networking:

- Social engineering and insider threat

- Decision-making in cyberspace

- Human-centric advances in cybersecurity

- Cross-cultural aspects of cybersecurity

- Behavioral and personality-based correlates of risk mitigation

- Emerging technologies and cyber literacy

- Information security awareness

All manuscripts should be submitted online using the manuscript submission portal by August 1, 2020. All submissions will be subject to a rigorous peer review. We encourage submissions of original research articles, reviews, and perspectives.

Visit Cyberpsychology, Behavior, and Social Networking online to learn more, read past issues, and view author submission guidelines.

\author{
Visit the Instructions for Authors: \\ www.liebertpub.com/cyber
}

\section{Submit your paper for peer review online: http://mc.manuscriptcentral.com/cyberpsych}

Mary Ann Liebert, Inc., publishers, 140 Huguenot Street, New Rochelle, NY 10801, USA. 